J. Synchrotron Rad. (1999). 6, 228-230

\section{Amplitude reduction in EXAFS}

\section{Roy* and S. J. Gurman}

Department of Physics, University of Leicester, Leicester LE1 7RH, U.K.England. E-mail: mr6@le.ac.uk

In real systems, inelastic processes remove photoelectrons from the elastic scattering channel. This reduces the amplitude of the EXAFS causing disagreement between the experimental and theoretically predicted amplitudes. Traditionally these discrepancies were treated by including two semi empirical reduction factors in the data analysis: a mean free path term, which models the so called extrinsic loss processes, and a constant amplitude reduction factor which accounts for many electron excitations at the absorbing atom. The extrinsic inelastic effects may, however, be modelled more rigorously using a complex exchange and correlation potential. For example the Hedin-Lundqvist (H-L) potential used in most EXAFS data analysis programs. We present a method by which the losses caused by such a potential may be evaluated quickly and easily in the first Born approximation. The losses produced by the H-L potential significantly overestimate those produced by the mean free path alone. Instead the losses appear to agree well with the total reduction given by the semi-empirical reduction factors. These losses do not exhibit the correct low or high energy behaviour but do show excellent agreement with experiment over the range of a typical EXAFS spectrum. We therefore conclude, that the semi-empirical reduction parameters should not be included when data fitting using the $\mathrm{H}-\mathrm{L}$ potential.

\section{Keywords: EXAFS; Many Body Theory; Inelastic processes.}

\section{Introduction}

Interpretation of the EXAFS may be based on a simple equation (Lee \& Pendry, 1975) obtained using the plane wave approximation. The single electron, single scattering expression for the EXAFS function $\chi(k)$ is,

$$
\begin{aligned}
\chi_{i}(k)= & \sum_{j} s_{o}^{2} e^{-2 R_{j} / \lambda(k)} e^{\left(-2 k^{2} \sigma_{j}^{2}\right)} \frac{N_{j}\left|f_{j}(k)\right|}{k R_{j}^{2}} \\
& \times \sin \left(2 k r_{j}+2 \delta_{j}(k, r)+\psi_{j}\right) .
\end{aligned}
$$

Equation (1) describes the EXAFS due to scattering by shells of $N_{j}$ atoms at a distance $R_{j}$ from the absorbing atom. $f_{j}(k)$ is the backscattering amplitude from each of the $N_{j}$ neighbours whilst the Debye-Waller factor, $\sigma_{j}$, allows for static and thermal disorder effects. The reduction factors, $e^{-2 R_{j} / \lambda(k)}$, and $s_{o}^{2}$ account for discrepancies between the predicted and experimentally measured amplitudes.

These discrepancies arise because only elastically scattered photoelectrons contribute to the primary channel EXAFS. With a real scattering potential, the reduction factors are needed to account for effective absorptions from the photoelectron beam due to various inelastic scattering processes. $e^{-2 R_{j} / \lambda(k)}$ is the mean free path term. This accounts for the extrinsic losses (Penn 1987), those produced by inelastic electron-electron scattering events at neighbouring atoms and in the medium in between. The constant amplitude reduction factor, $s_{o}^{2}$, approximates the losses due to multiple electron excitations at the absorbing atom. The multiple electron excitations are caused by the creation of a core hole in addition to the photoelectron when the $\mathrm{x}$-ray photon is absorbed. They are usually referred to as the 'intrinsic losses' in the literature (Bardyszewski \& Hedin, 1985).

Most modern EXAFS data analysis programs use a complex exchange and correlation potential to model the extrinsic inelastic effects, for example the H-L potential (Hedin \& Lundqvist, 1969) used in the standard Daresbury package EXCURV92 (Binsted $e t a l$, 1992). The H-L potential approximates the effect of the electronelectron interactions as the photoelectron propagates between the central and neighbouring atoms. This potential gives rise to complex phase shifts in equation (1) and therefore reduces the amplitude of the EXAFS without the need for ad hoc reduction parameters. We shall now proceed to calculate the amplitude of the EXAFS in the presence of the H-L potential.

\section{Calculation}

We could, in principle, use a program such as EXCURV92 to investigate the effect of the H-L potential on the EXAFS amplitudes. The full EXAFS could be calculated both in the presence and the absence of the imaginary part of the $\mathrm{H}-\mathrm{L}$ potential and the amplitudes compared. However for the sake of ease of computation we choose to evaluate the EXAFS in the first Born approximation. This approach gives the standard textbook results in the case of an isolated atom (Bransden and Joachain 1983).

Standard single electron EXAFS theory may be developed within the 'muffin tin' approximation using the Hartree photoelectron Greens function, $G_{c}\left(r, r^{\prime}\right)$, and the basis state wave function, $\phi_{o}(k r)$. Where $\phi_{o}(k r)=\sum_{l m} 2 \pi \imath^{l} e^{i \delta_{l}} R_{l}(k r) Y_{l m}(\hat{r}) Y_{l m}^{*}(\hat{k})$ inside the muffin tin, and $R_{l}(k r)$ is a regular solution of the Schrödinger equation in the presence of the atomic potential and the real part of the $\mathrm{H}-\mathrm{L}$ potential. We examine the effect of the imaginary part of the H-L potential, $V_{I}$, on the EXAFS by treating it as a perturbation on the wave functions, $\phi_{o}(k r) . V_{I}$ is zero outside each of the muffin tin spheres. To first order in $V_{I}$, $\phi_{1}(k r)=\phi_{o}(k r)+G_{c}\left(r, r^{\prime}\right) V_{I}\left(r^{\prime}\right) \phi_{o}(k r)$. Outside the muffin tin the perturbed basis state is then found to have the same functional form as $\phi_{o}(k r)$. The phase-shifted parts are merely multiplied by a factor $\left\{1+\frac{k m}{\hbar^{2}}\left\langle R_{l}\left|V_{l}\right| R_{l}\right\rangle\right\}$ which we can subsume into the phase shifts making $\delta_{l}$ complex. Using the complex phase shifts, $\delta_{l}^{\prime}=\delta_{l}+\frac{\imath k m}{2 \hbar^{2}}\left\langle R_{l}\left|V_{l}\right| R_{l}\right\rangle$, in the expression for the basis state wave functions and in the Hartree Greens function we may proceed with the standard theoretical treatment to derive the EXAFS in a form similar to equation (1),

$$
\begin{aligned}
\chi_{i}(k)= & \sum_{j} e^{\left(-2 k^{2} \sigma_{j}^{2}\right)} \frac{N_{j}\left|f_{j}^{\prime}(k)\right|}{k R_{j}^{2}}\left\{1-\frac{2 k m}{\hbar^{2}}\left\langle R_{l}\left|V_{I}\right| R_{l}\right\rangle\right\} \\
& \times \sin \left(2 k r_{j}+2 \delta_{j}(k, r)+\psi_{j}\right) .
\end{aligned}
$$

In equation (2) $l$ is the angular momentum of the relevant photoelectron final state and the perturbed backscattering factor, $f_{j}^{\prime}(k)$, is given by,

$f_{j}^{\prime}(k)=\frac{\imath}{2 k} \sum_{L}(-1)^{L}(2 L+1)\left\{1-\frac{k m}{\hbar^{2}}\left\langle R_{L}\left|V_{I}\right| R_{L}\right\rangle e^{2 \imath \delta_{L}}\right\}$,

Comparing equations ( 1 ) and (2) we can easily see that the amplitude reduction produced by the imaginary part to the $\mathrm{H}-\mathrm{L}$ potential is given by,

$$
A=\left\{1-\frac{2 k m}{\hbar^{2}}\left\langle R_{l}\left|V_{I}\right| R_{l}\right\rangle\right\} \frac{\left|f_{j}^{\prime}(k)\right|}{\left|f_{j}(k)\right|}
$$


We calculate the radial wave functions used in equation (4) by numerically integrating out the Schrödinger equation to the edge of the muffin tin sphere. The calculated phase shifts are then used to evaluate $f_{j}(k)$ and $f_{j}^{\prime}(k)$.

The exchange and correlation potential is also evaluated numerically. We follow a scheme outlined by Lee and Beni (Lee \& Beni, 1976) who first developed the H-L potential for EXAFS calculations by applying standard results for the electron self energy in a homogeneous electron gas within a local density formalism. The self energy is calculated using the single plasmon pole approximation in which the elementary excitations of the electron gas are replaced by a single plasmon-like absorption for all wavevectors. This absorption is weighted so as to exhaust the sum rule on the dielectric function (Hedin \& Lundqvist, 1969). Thus the H-L potential should include all the relevant inelastic electron-electron scattering events.

\section{Results}

The EXAFS amplitude is calculated using equation (4) for three elements: Silicon, Copper and Silver. This is then compared to known values of the empirical parameters, $s_{o}^{2}(k)$ and $e^{-2 R_{j} / \lambda(k)}$, fitted using the real X-alpha potential for the three elements with the data analysis program EXCURV92. The best fit values of $s_{o}^{2}$ and an effective, constant, imaginary potential, $V_{P I}$, were found to be $s_{o}^{2}=0.72 \pm 0.1$ for Silicon, $s_{o}^{2}=0.70 \pm 0.1$ for Copper, and $s_{o}^{2}=0.67 \pm 0.15$ for Silver. The $V_{P I}$ values used were: $-4.0 \mathrm{eV} \pm 0.2 \mathrm{eV}$ for Silicon, $-4.0 \mathrm{eV} \pm 0.2 \mathrm{eV}$ for Copper and $-6.3 \mathrm{eV} \pm 0.2 \mathrm{eV}$ for Silver where the quoted uncertainties are $2 \sigma$ errors. The mean free path term is calculated from the constant imaginary potential, $V_{P I}$, using the standard relation $\lambda(k)=\hbar^{2} k / m V_{P I}$.

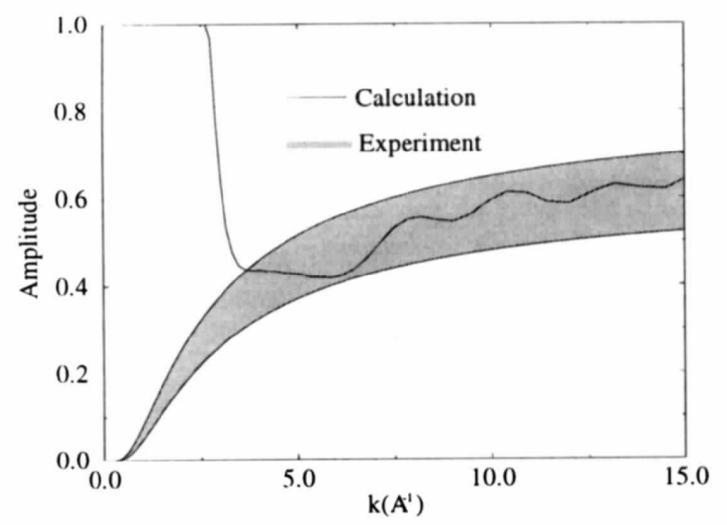

Figure 1

Calculated and best fit EXAFS reduction factors plotted against photoelectron wave vector for Silicon.

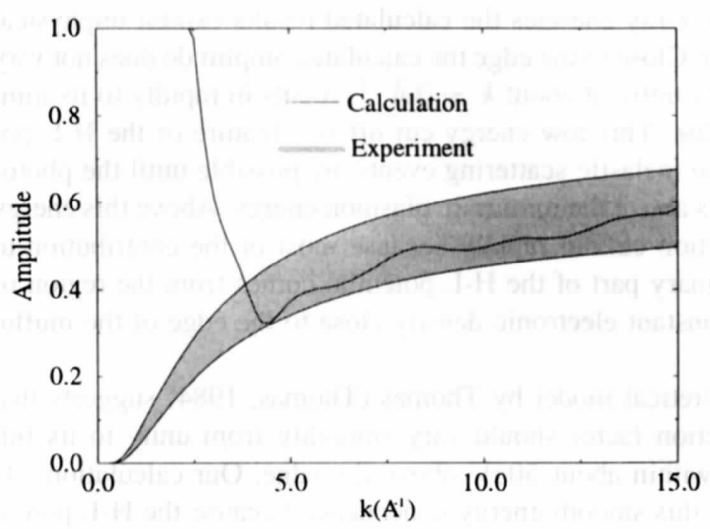

Figure 2

Calculated and best fit EXAFS reduction factors plotted against photoelectron wave vector for Copper.

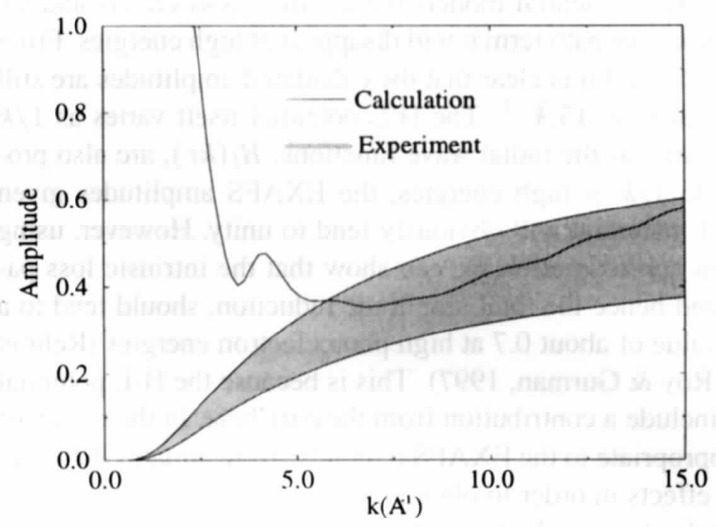

Figure 3

Calculated and best fit EXAFS reduction factors plotted against photoelectron wave vector for Silver.

In figures 1, 2 and 3 we plot the calculated amplitude as a function of the photoelectron wave-vector, $k$, in $\AA^{-1}$. The solid band shows the product of the fitted values of $s_{o}^{2}$ and $e^{-2 R_{j} / \lambda(k)}$ within the limit of experimental error. We can clearly see that the losses obtained from the H-L potential agree with the total reduction given by the semi-empirical reduction parameters and must therefore overestimate that produced by the mean free path effects alone. Over the range of a typical EXAFS spectrum, $4 \AA^{-1}$ to $14 \AA^{-1}$, the calculated reduction parameters lie within the error range of the best fit experimental data. We measure the correlation between our calculations and the fitted empirical parameters in this region using the R-factor, $R=\frac{\sum \mid \text { expt-theory } \mid}{\sum \mid \text { expt } \mid} \times 100 \%$. We find $R=4.9 \%$ for Silicon, $R=6.5 \%$ for Copper, and $R=10.4 \%$ for Silver. These uncertainties are much lower than those observed in the best fit parameters for the various elements: $15.5 \%$ for Silicon, $16.1 \%$ for Copper and $24.4 \%$ for Silver, which suggests that the reduction given by the H-L potential is equivalent to that produced by the semi-empirical reduction parameters.

EXCURV92 was also used to fit values for the adjustable parameter, $s_{o}^{2}$, using the H-L potential. We found values of $s_{o}^{2}(k)=$ $0.92 \pm 0.10$ for Silicon, $s_{o}^{2}(k)=1.05 \pm 0.05$ for Copper and $s_{o}^{2}(k)=1.02 \pm 0.05$ for Silver. These values are all consistent with unity which again would suggest that, empirically, the H-L potential accounts for all of the losses to the EXAFS. 
At low $\mathrm{x}$-ray energies the calculated results exhibit unphysical behaviour. Close to the edge the calculated amplitude does not vary from unity until, at about $k \sim 3 \AA^{-1}$, it cuts in rapidly to its minimum value. This low energy cut off is a feature of the H-L potential. No inelastic scattering events are possible until the photoelectron is above the minimum plasmon energy. Above this energy the reduction cuts in rapidly because most of the contribution to the imaginary part of the H-L potential comes from the region of almost constant electronic density close to the edge of the muffin tin.

A theoretical model by Thomas (Thomas, 1984) suggests that the reduction factor should vary smoothly from unity to its full strength within about $50 \mathrm{eV}$ above the edge. Our calculations do not show this smooth energy dependence because the H-L potential does not include single electron excitations with energies below the minimum plasmon energy. At higher energies these single electron events are included by appropriately weighting the plasmon excitation strength, thus this failure only occurs at low $k$.

At high $k$ the $\mathrm{H}$-L potential overestimates the EXAFS amplitudes. The $\mathrm{H}-\mathrm{L}$ potential models the extrinsic loss effects and so, like the mean free path term it will disappear at high energies. From Figures 1, 2 and 3 it is clear that the calculated amplitudes are still increasing at $k=15 \AA^{-1}$. The $\mathrm{H}$-L potential itself varies as $1 / k$ at large $\mathrm{k}$, and, as the radial wave functions, $R_{l}(k r)$, are also proportional to $1 / k$ at high energies, the EXAFS amplitudes given by the $\mathrm{H}-\mathrm{L}$ potential will obviously tend to unity. However, using the sudden approximation we can show that the intrinsic loss parameter, and hence the total amplitude reduction, should tend to a constant value of about 0.7 at high photoelectron energies (Rehr et $a l, 1978$, Roy \& Gurman, 1997). This is because the H-L potential does not include a contribution from the core hole. In the region of energy appropriate to the EXAFS it merely overestimates the mean free path effects in order to obtain the correct amplitude reduction factors. Indeed, an explicit calculation of the mean free path using the $\mathrm{H}-\mathrm{L}$ potential gives values much shorter than those observed experimentally.

\section{Conclusion}

We have calculated the effect of the imaginary part of the H-L potential on the EXAFS amplitude using a perturbation series to first order in $V_{I}(r)$. We find that the H-L potential overestimates the losses to the EXAFS due to the finite photoelectron mean free path alone. However, it happens to give excellent agreement with the total experimental losses in the range of most EXAFS data analysis.

Empirically the H-L potential accounts for all of the losses to the EXAFS amplitudes. We therefore conclude that it should not be necessary to use the additional amplitude fitting parameters, $s_{o}^{2}$ and $e^{-2 R_{j} / \lambda(k)}$, when data fitting with the H-L potential.

However the $\mathrm{H}-\mathrm{L}$ potential is 'merely a phenomenological model that happens to work for the EXAFS' (Tyson 1991). It also does not exhibit the correct behaviour at very high and low photoelectron energies. As such the development of a more accurate exchange and correlation potential for use in EXAFS calculations would be desirable.

\section{References}

P A Lee \& J B Pendry, Phys. rev: B 11 (1975) 2785-2811

W Bardyszewski \& L Hedin, Physica Scripta, 32 (1985) 439-450

L Hedin \& S Lundqvist, Solid State Physics, 23 (1969) 2-181

D R Penn, Phys Rev B, 35 (1987) 482-486

N Binsted, S J Gurman, T C Campbell, \& P C Stephenson, EXCURV92 program (Daresbury: SERC Daresbury Laboratory) 1992

B H Bransden \& C J Joachain Physics of Atoms and Molecules, Longman Scientific and Technical, (1983), 494

P A Lee \& G Beni, Phys. Rev. B 15 (1976), 2862-2883

T Darrah Thomas, Phys. Rev. Letters 52 (1984) 417-420

J J Rehr, E A Stern, R L Martin \& E A Davidson, Phys. Rev: B17(1978), 560-565

M Roy \& S J Gurman, J. Phys. IV France Colloque C2 151-152

T A Tyson, PhD Thesis. Stanford University (1991)

(Received 10 August 1998; accepted 9 December 1998) 